\title{
PHOTON-DOMINATED AND X-RAY DOMINATED REGIONS
}

\section{A. STERNBERG}

School of Physics and Astronomy

Tel Aviv University, Ramat Aviv 69978

Tel Aviv, ISRAEL

AND

MIN YAN AND A. DALGARNO

Harvard-Smithsonian Center for Astrophysics

60 Garden Street

Cambridge, Massachusetts, 02138 USA

\begin{abstract}
A brief account is presented of the chemical characteristics of regions controlled by the absorption of X-rays. Recent studies of the infrared emission from molecular hydrogen and time-dependent effects in evolving photon-dominated regions are summarized.
\end{abstract}

\section{Introduction}

Photon-dominated regions or photodissociation regions (PDRs) are regions of gas exposed to far ultraviolet radiation. PDRs occur at the boundaries of HII regions and planetary and reflection nebulae, on the edges of and inside clumpy molecular clouds, in starburst galaxies and in the narrow line regions of active galactic nuclei (Stacey et al. 1993, Van der Werf et al. 1993, 1996, Zhou et al. 1993, Störzer, Stutzki \& Sternberg 1996, Luhman \& Jaffe 1996, Luhman \& Rieke 1996). Because of absorption by $\mathrm{H}_{2}$ and by grains, PDRs are limited in spatial extent and they exist as narrow interfaces, separating ionized and neutral material.

$\mathrm{X}$-ray dominated regions or X-ray dissociation regions (XDRs) are regions of gas exposed to X-rays. XDRs occur in molecular clouds with embedded X-ray sources, in active galactic nuclei and near to supernova remnants and high velocity shocks. X-ray absorption cross sections diminish 
rapidly at high photon frequencies and XDRs may extend over large spatial dimensions.

In PDRs, molecular formation is driven by photoionization and molecular destruction by photodissociation. In XDRs, molecular formation is again driven by photoionization but destruction occurs through ion-molecule reactions and by the secondary effects of the energetic photoelectrons, including photodissociation by the absorption of internally generated ultraviolet photons.

Both PDRs and XDRs contain zones of enhanced temperature in which endothermic reactions with $\mathrm{H}_{2}$ contribute significantly to molecular formation. Both PDRs (Black \& Dalgarno 1976) and XDRs (Lepp \& McCray 1983) respond to the external radiation field by exciting vibrational levels of molecular hydrogen $\mathrm{H}_{2}{ }^{*}$. The presence of $\mathrm{H}_{2}{ }^{*}$ enhances molecular formation (Sternberg \& Dalgarno 1995) and it gives rise to a many-lined infrared emission spectrum. The spectrum offers a powerful diagnostic probe of the nature of the incident radiation field and a reliable measure of its intensity. Other strong atomic and molecular emission lines at infrared and microwave frequencies are produced which provide additional probes of the regions (Tielens \& Hollenbach 1985, van Dishoeck \& Black 1988, Sternberg \& Dalgarno 1989, Hollenbach, Takahashi \& Tielens 1991, Escalante, Sternberg \& Dalgarno 1991, Meixner \& Tielens 1993, 1995, Natta, Walmsley \& Tielens 1994).

\section{Chemistry in dense PDRs}

The physical and chemical properties of PDRs are controlled by the incident photons which penetrate the clouds, heat the gas and drive the cloud chemistry. The cloud structure depends on the gas density and pressure, the intensity of the far ultraviolet radiation field, the absorption and scattering properties of the dust grains, the element abundances and the cloud geometry (Tielens \& Hollenbach 1985, van Dishoeck \& Black 1988, Le Bourlot et al. 1993, Köster et al. 1994, Störzer, Stutzki \& Sternberg 1995). In dense clouds which fully absorb the incident radiation, the size of the PDRs is set by the dust opacity to cloud depths corresponding to visual extinctions $A_{V}$ of a few magnitudes. Detailed model calculations (Sternberg \& Dalgarno 1995, Jansen et al. 1995) show that in a PDR photoionization and charge transfer processes give rise to a layered ionization structure. Fig. 1 illustrates the structure for a cloud of density $2 \times 10^{5} \mathrm{~cm}^{-3}$. Beyond the $\mathrm{H} / \mathrm{H}_{2}$ transition region which lies adjacent to the ultraviolet source is a zone in which $\mathrm{C}^{+}$is the major ion. The molecular ions $\mathrm{HCO}^{+}, \mathrm{H}_{3} \mathrm{O}^{+}$and $\mathrm{H}_{3}^{+}$take over in the dark core where cosmic rays are the principal ionizing agent. For a density of $1 \times 10^{6} \mathrm{~cm}^{-3}$ and a radiation field intensity $2 \times 10^{5}$ times 


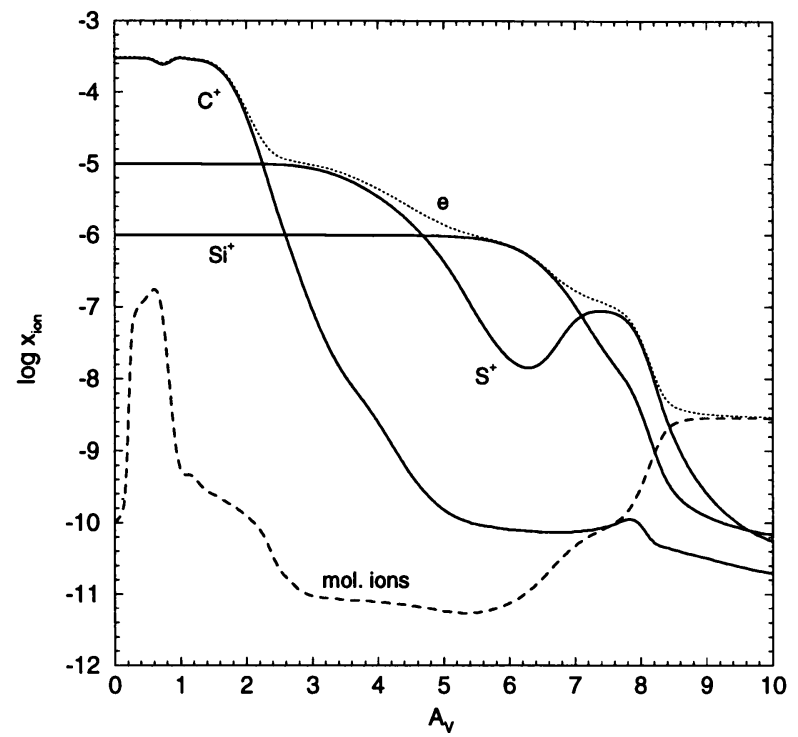

Figure 1. The ionization structure of a photon-dominated region

the mean interstellar value, the $\mathrm{S}^{+}$zone begins at a visual magnitude $A_{V}$ of 2 and the $\mathrm{Si}^{+}$at $A_{V}$ between 4 and 5 . The dark core begins at an $A_{V}$ near $8 \mathrm{mag}$. Ambipolar diffusion rates and magnetically controlled collapse times are sensitively dependent on the fractional ionization in the different cloud layers (McKee 1989, Hartquist et al. 1993).

In the transition from the $\mathrm{C}^{+}$zone to the $\mathrm{S}^{+}$zone, $\mathrm{C}^{+}$is converted by ion-molecule reactions to $\mathrm{CO}$ and to $\mathrm{CH}$. Photodissociation then enhances the abundance of neutral carbon atoms. PDRs at the surfaces of molecular cloud clumps may explain the observed high $\mathrm{C} / \mathrm{CO}$ ratios as the dissociating ultraviolet radiation penetrates the interclump gas (Phillips \& Huggins 1981, Keene et al. 1985, Stutzki et al. 1988, Genzel et al. 1988, Zmuidzinas et al. 1988, Schilke et al. 1993, Tauber et al. 1995). A high fractional abundance of neutral carbon atoms can also be created by recombination of $\mathrm{C}^{+}$ions in a cooling ionized region that is shadowed by a cloud (Störzer, Stutzki \& Sternberg 1996).

\section{1. $\mathrm{OH}, \mathrm{CO}^{+}, \mathrm{AND} \mathrm{SO}^{+}$}

In dense clouds with hydrogen nuclei densities $n_{\mathrm{H}}$ greater than $10^{3} \mathrm{~cm}^{-3}$ the outer edges are hot with temperatures in excess of $10^{3}$. The gas cools rapidly as atomic hydrogen is converted to $\mathrm{H}_{2}$. Once some $\mathrm{H}_{2}$ has formed, $\mathrm{OH}$ is readily produced by the reactions

$$
\mathrm{O}+\mathrm{H}_{2} \rightarrow \mathrm{OH}+\mathrm{H}
$$


and

$$
\mathrm{O}+\mathrm{H}_{2}^{*} \rightarrow \mathrm{OH}+\mathrm{H} \text {. }
$$

The hydroxyl radical is removed by photodissociation

$$
\mathrm{OH}+\nu \rightarrow \mathrm{O}+\mathrm{H}
$$

and by the reverse of reaction (1). Large fractional abundances of $\mathrm{OH}$ can be achieved. In a cloud with $n_{\mathrm{H}}=10^{6} \mathrm{~cm}^{-3}$ and an ultraviolet radiation field a factor of $2 \times 10^{5}$ more intense than the mean interstellar field the peak $\mathrm{OH}$ fractional abundance is $10^{-6}$. The $\mathrm{OH}$ reacts with the atoms and ions produced by photodissociation and photoionization. The molecular ions $\mathrm{CO}^{+}, \mathrm{SO}^{+}$and $\mathrm{SiO}^{+}$are formed by

$$
\begin{gathered}
\mathrm{C}^{+}+\mathrm{OH} \rightarrow \mathrm{CO}^{+}+\mathrm{H}, \\
\mathrm{S}^{+}+\mathrm{OH} \rightarrow \mathrm{SO}^{+}+\mathrm{H}
\end{gathered}
$$

and

$$
\mathrm{Si}^{+}+\mathrm{OH} \rightarrow \mathrm{SiO}^{+}+\mathrm{H}
$$

and removed by dissociative recombination, photodissociation and reactions with $\mathrm{H}_{2}$. Neutral molecules $\mathrm{O}_{2}$ and $\mathrm{NO}$ are formed by

$$
\mathrm{O}+\mathrm{OH} \rightarrow \mathrm{O}_{2}+\mathrm{H}
$$

and

$$
\mathrm{N}+\mathrm{OH} \rightarrow \mathrm{NO}+\mathrm{H}
$$

and removed by photodissociation. Water is produced by

$$
\mathrm{OH}+\mathrm{H}_{2} \rightarrow \mathrm{H}_{2} \mathrm{O}+\mathrm{H}
$$

and lost by the reverse reaction to (9) and by photodissociation.

The molecular ion $\mathrm{CO}^{+}$attains a fractional abundance of $10^{-9}$ in the $\mathrm{H} / \mathrm{H}_{2}$ transition layer and disappears at greater depths. Observational evidence for the production of $\mathrm{CO}^{+}$in hot PDRs has been obtained by the detection of rotational emission lines of $\mathrm{CO}^{+}$at 236.06 and $235.79 \mathrm{GHz}$ in the planetary nebula NGC 7027 and the molecular cloud interfaces in the Orion Bar and M17 (Latter, Walker \& Maloney 1995, Störzer, Stutzki \& Sternberg 1995). The observations indicate that the emissions peak at the edges of the molecular clouds near but outside the ionization fronts, decreasing sharply in intensity with depth into the clouds (Störzer et al. 1995). The measured line intensities indicate a column density of $\mathrm{CO}^{+}$of nearly $10^{13} \mathrm{~cm}^{-2}$, an order of magnitude larger than the theoretical prediction for a PDR. The Orion Bar and M17 are edge-on systems and the optically thin emission may be enhanced by limb brightening. 
The ion $\mathrm{CO}^{+}$is also produced in dissociative shocks (Neufeld \& Dalgarno 1989) so that its presence alone does not establish a PDR as its origin. Dissociative shocks and PDRs may be distinguished by observations of $\mathrm{SO}^{+}$ and $\mathrm{SO} . \mathrm{SO}^{+}$has been detected in many astrophysical sources (Turner 1992, 1994). It is produced in dissociative shocks (Neufeld \& Dalgarno 1989) and Turner $(1992,1994)$ has argued that the presence of $\mathrm{SO}^{+}$in shocked gas is strong evidence that the shock is dissociative. It is also produced in PDRs. The fractional abundance of $\mathrm{SO}^{+}$in the $\mathrm{H} / \mathrm{H}_{2}$ transition zone is $7 \times 10^{-10}$ in the model of Sternberg \& Dalgarno (1995) and a column density of $5 \times 10^{11} \mathrm{~cm}^{-2}$ is predicted for a sulfur fractional abundance of $10^{-5}$. The same model predicts a low abundance of $\mathrm{SO}$ in contrast to the dissociative shock models in which $\mathrm{SO}$ and $\mathrm{SO}^{+}$have comparable abundances (Neufeld \& Dalgarno 1989).

\subsection{CN/HCN DENSITY RATIOS}

Another diagnostic probe of PDRs is the CN/HCN density ratio (Fuente et al. 1993, Sternberg \& Dalgarno 1995). HCN is created by the reactions

$$
\begin{gathered}
\mathrm{H}_{2} \mathrm{CN}^{+}+e \rightarrow \mathrm{HCN}+\mathrm{H} \\
\mathrm{HCN}^{+}+\mathrm{H} \rightarrow \mathrm{HCN}+\mathrm{H}^{+} \\
\mathrm{CN}+\mathrm{H}_{2} \rightarrow \mathrm{HCN}+\mathrm{H} \\
\mathrm{CH}_{2}+\mathrm{N} \rightarrow \mathrm{HCN}+\mathrm{H} \\
\mathrm{CH}_{3}+\mathrm{N} \rightarrow \mathrm{HCN}+\mathrm{H}_{2}
\end{gathered}
$$

with varying efficiencies in the different zones of PDRs. Its photodissociation

$$
\mathrm{HCN}+\nu \rightarrow \mathrm{CN}+\mathrm{H}
$$

is a major source of CN. Fig. 2 presents calculations of the equilibrium $\mathrm{CN} / \mathrm{HCN}$ density ratio for PDRs of densities $n_{\mathrm{H}}$ greater than or equal to $10^{3} \mathrm{~cm}^{-3}$ subjected to a radiation field intensity of $2 \times 10^{5}$ times the interstellar value. Fuente et al. $(1993,1995)$ found enhanced CN/HCN ratios in the reflection nebulae NGC 7023 and NGC 2023. The observed ratios decreased sharply by a factor of seven in NGC 7023 and twenty-five in NGC 2023 with increasing distances from the illuminating stars. In NGC 2023, high density $\mathrm{CN}$ filaments coincide in position with infrared emission from vibrationally excited $\mathrm{H}_{2}$.

\subsection{CS IN NEUTRAL SULFUR ZONES}

The CS molecule is often employed as a diagnostic probe of dense gas. It may also be a probe of molecular clouds, illuminated by far ultraviolet 


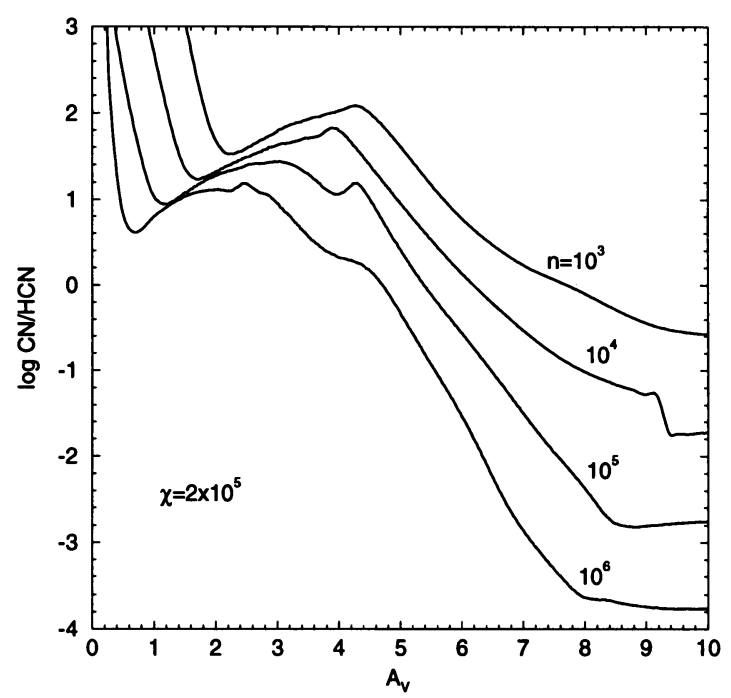

Figure 2. The $\mathrm{CN} / \mathrm{HCN}$ abundance ratio in a PDR with densities $n_{\mathrm{H}}$ greater than or equal to $10^{3} \mathrm{~cm}^{-3}$.

radiation. Models (Sternberg \& Dalgarno 1995) show an enhancement of CS in the neutral sulfur zone at intermediate cloud depths compared to fully shielded regions. Some $\mathrm{S}^{+}$exists in the S I zone and the sequence

$$
\begin{gathered}
\mathrm{S}^{+}+\mathrm{H}_{2} \rightarrow \mathrm{SH}_{2}^{+}+\nu \\
\mathrm{SH}_{2}^{+}+e \rightarrow \mathrm{SH}+\mathrm{H} \\
\mathrm{SH}+\mathrm{O} \rightarrow \mathrm{SO}+\mathrm{H} \\
\mathrm{SO}+\mathrm{C} \rightarrow \mathrm{CS}+\mathrm{O}
\end{gathered}
$$

produces CS. The $\mathrm{S}^{+}$ions and carbon atoms are the result of the cosmic ray ionization of helium, followed by

$$
\mathrm{He}^{+}+\mathrm{CO} \rightarrow \mathrm{He}+\mathrm{C}^{+}+\mathrm{O}
$$

and

$$
\mathrm{C}^{+}+\mathrm{S} \rightarrow \mathrm{C}+\mathrm{S}^{+} \text {. }
$$

The CS molecules are removed by photodissociation. The peak in the CS density occurs at the inner edge of the S I zone where the far ultraviolet field is attenuated but most of the sulfur is in atomic form. At larger depths, the density of $\mathrm{S}$ atoms decreases as sulfur is incorporated into $\mathrm{SO}$ and $\mathrm{SO}_{2}$. 


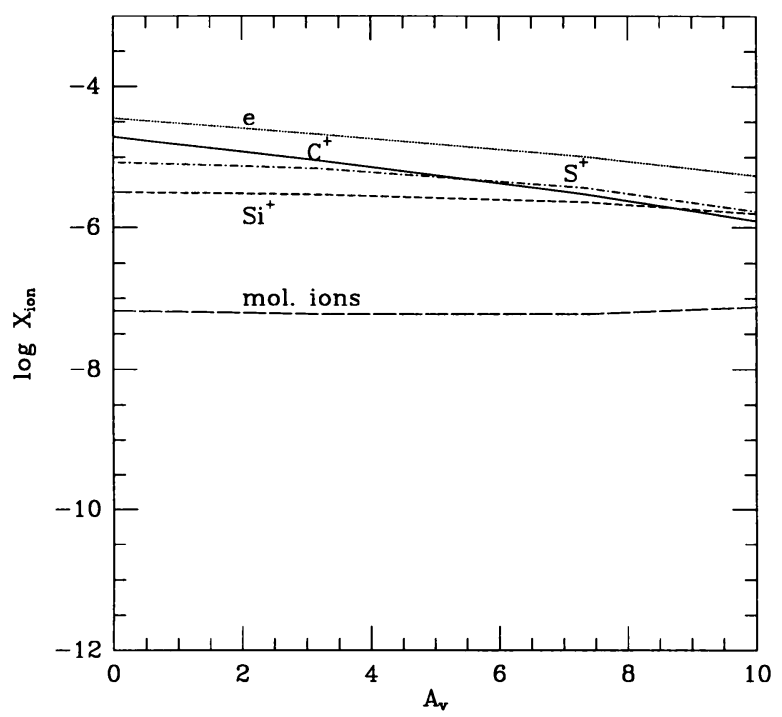

Figure 3. The ionization structure of an X-ray dominated region

In the model of Sternberg \& Dalgarno (1995) the CS density is a factor of $10^{3}$ larger at the inner edge of the S I zone than in the dark molecular core.

\section{Chemistry of XDRs}

The molecular composition of XDRs depends on the intensity of the incident X-rays. Initially, the increased ionization rate accelerates the formation of molecules and molecular abundances are enhanced (Krolik \& Kallman 1983) but ultimately the destructive influence of the X-rays dominates (Lepp \& Dalgarno 1996). In Fig. 3 we present the ionization structure of an XDR in which the unattenuated ionization rate is $2.5 \times 10^{-13} \mathrm{~s}^{-1}$ and the density is $2 \times 10^{5} \mathrm{~cm}^{-3}$. The ionization is dominated by $\mathrm{H}^{+}$and $\mathrm{He}^{+}$. The chemistry follows the formation and destruction of molecular hydrogen. In the warm zone, the chemistry of hydrogen is similar to that prevailing in the early Universe. Molecular hydrogen is produced by radiative association

$$
\mathrm{H}+\mathrm{H}^{+} \rightarrow \mathrm{H}_{2}^{+}+\nu
$$

followed by

$$
\mathrm{H}_{2}^{+}+\mathrm{H} \rightarrow \mathrm{H}_{2}+\mathrm{H}^{+}
$$

and radiative attachment

$$
e+\mathrm{H} \rightarrow \mathrm{H}^{-}+\nu
$$


followed by associative detachment

$$
\mathrm{H}+\mathrm{H}^{-} \rightarrow \mathrm{H}_{2}+e .
$$

The presence of helium enhances the formation of $\mathrm{H}_{2}$ through the sequence

$$
\begin{gathered}
\mathrm{H}^{+}+\mathrm{He} \rightarrow \mathrm{HeH}^{+}+\nu \\
\mathrm{He}^{+}+\mathrm{H} \rightarrow \mathrm{HeH}^{+}+\nu \\
\mathrm{HeH}^{+}+\mathrm{H} \rightarrow \mathrm{H}_{2}^{+}+\mathrm{He}
\end{gathered}
$$

and reaction (23). The presence of $\mathrm{HeH}^{+}$is a potential diagnostic (Roberge \& Dalgarno 1982, Maloney, Hollenbach \& Tielens 1996). The $\mathrm{H}_{2}$ may be vibrationally excited, in which case it can be removed by charge transfer with $\mathrm{He}^{+}$

$$
\mathrm{He}^{+}+\mathrm{H}_{2}^{*} \rightarrow \mathrm{He}+\mathrm{H}+\mathrm{H}^{+}
$$

and by dissociative attachment

$$
e+\mathrm{H}_{2}^{*} \rightarrow \mathrm{H}+\mathrm{H}^{-} .
$$

In the cold zone, $\mathrm{H}_{2}$ is formed primarily by surface catalysis. It is destroyed in the warm zone by collisional dissociation in neutral particle collisions,

$$
\mathrm{H}+\mathrm{H}_{2} \rightarrow \mathrm{H}+\mathrm{H}+\mathrm{H}^{+}
$$

and by electron impact excitation to the dissociative $b^{3} \Sigma_{u}^{+}$state,

$$
\begin{aligned}
e+\mathrm{H}_{2} & \rightarrow e+\mathrm{H}_{2}\left(b^{3} \Sigma_{u}^{+}\right) \\
& \rightarrow e+\mathrm{H}+\mathrm{H} .
\end{aligned}
$$

The abundance of $\mathrm{H}_{2}$ is reduced also through photoionization by photons emitted following radiative recombination of $\mathrm{He}^{+}$ions

$$
\mathrm{He}^{+}+e \rightarrow \mathrm{H}+\nu
$$

and following excitation of electronic states of $\mathrm{He}$

$$
\begin{gathered}
e+\mathrm{He} \rightarrow \mathrm{He}^{*}+e \\
\mathrm{He}^{*} \rightarrow \mathrm{He}+\nu .
\end{gathered}
$$

In XDRs the high frequency X-rays are preferentially absorbed by heavy elements, as in the interstellar gas in inner shell transitions (Morrison \& McCammon 1983). The ejection of the innershell electrons is followed by Auger 
and radiative Auger decay processes leading to the creation of multiplycharged ions (Weisheit \& Dalgarno 1972, Weisheit 1974). The ionization of the light elements $\mathrm{H}, \mathrm{H}_{2}$ and $\mathrm{He}$ arises from collisions of the energetic photoelectrons

$$
e+\left(\mathrm{H}, \mathrm{H}_{2}, \mathrm{He}\right) \rightarrow e+\left(\mathrm{H}^{+}, \mathrm{H}_{2}^{+}, \mathrm{He}^{+}\right)+e
$$

but the light elements remain partly neutral beyond the fully-ionized zone that lies close to the ionizing source (Lepp \& McCray 1983). Neutral hydrogen and helium and multiply-charged heavy ions co-exist. The multiplycharged ions undergo sequential charge transfer. Most are quickly reduced to singly charged ions which enter a chemistry much like that driven by cosmic ray ionization. For some doubly-charged ions, charge transfer may be slow. The doubly-charged ions $\mathrm{C}^{2+}$ and $\mathrm{S}^{2+}$ may be signatures of XDRs or they may react with $\mathrm{H}_{2}$ producing $\mathrm{CH}^{+}$(Dalgarno 1976, Langer 1978) and $\mathrm{SH}^{+}$.

Photodissociation of molecules by ultraviolet photons generated internally by the photoelectrons as they slow down in the gas (Prasad \& Tarafdar 1983) is an important mechanism for the destruction of molecules, as are reactions with $\mathrm{He}^{+}$and $\mathrm{H}^{+}$. The dissociation products may participate in reactions to form other molecules. Diatomic molecules, particularly hydrides, are resistant to destruction and can exist in considerable abundance in the presence of intense X-ray radiation (Lepp \& Dalgarno 1996). Detailed models of XDRs have been constructed by Maloney, Hollenbach \& Tielens (1996) and by Yan \& Dalgarno (1996a).

\section{1. $\mathrm{OH}$ AND $\mathrm{H}_{2} \mathrm{O}$}

The chemistry of the X-ray heated zone is similar to that summarized in $\S 2.1$. The molecules $\mathrm{OH}$ and $\mathrm{H}_{2} \mathrm{O}$ are produced in abundance (Lepp \& McCray 1983). Neufeld, Maloney \& Conger (1994) and Neufeld \& Maloney (1995) have argued that water maser emission associated with active galactic nuclei is a result of collisional pumping in dense gas heated by the absorption of X-rays.

\section{2. $\mathrm{HCO}^{+}$}

The production of $\mathrm{HCO}^{+}$in gas irradiated by X-rays has been discussed by Wolfire \& Königl (1993) in a model of the emission from Herbig-Haro objects and by Lepp \& Dalgarno (1996) and Yan \& Dalgarno (1996b) in a model of the molecular cloud that may surround the hard X-ray source $1 \mathrm{E}$ 1740.7-2942. The $\mathrm{HCO}^{+}$is produced in the warm zone by the reactions

$$
\mathrm{C}^{+}+\mathrm{OH} \rightarrow \mathrm{CO}^{+}+\mathrm{H}
$$




$$
\begin{gathered}
\mathrm{CO}^{+}+\mathrm{H}_{2} \rightarrow \mathrm{HCO}^{+}+\mathrm{H} \\
\mathrm{O}+\mathrm{CH} \rightarrow \mathrm{HCO}^{+}+e
\end{gathered}
$$

and deeper into the cloud by

$$
\mathrm{H}_{3}^{+}+\mathrm{CO} \rightarrow \mathrm{HCO}^{+}+\mathrm{H}_{2} .
$$

Phillips \& Lazio (1995) carried out interferometric observations of $\mathrm{HCO}^{+}$ and found that the emission peak is displaced from the source. They attributed the offset to the enhanced destruction and reduced formation of $\mathrm{HCO}^{+}$close to the source.

\section{Infrared emission of $\mathbf{H}_{2}$}

A powerful diagnostic probe of PDRs and XDRs is provided by the infrared emission from rovibrational levels of molecular hydrogen. Ultraviolet photons are absorbed into the excited $\left(B^{1} \Sigma_{u}^{+}\right)$and $\left(C^{1} \Pi_{u}\right)$ states which cascade into the rovibrational levels of the ground state (Gould \& Harwit 1963, Black \& Dalgarno 1976). The resulting infrared and visible spectra have been calculated in considerable detail (Black \& Dalgarno 1976, Black \& van Dishoeck 1987, Sternberg 1988, Sternberg \& Dalgarno 1989, Draine \& Bertoldi 1996, Neufeld \& Spaans 1996). Particularly clear examples of PDRs are the reflection nebula NGC 2023 (Gatley et al. 1987, Hasegawa et al. 1987, Burton et al. 1992, Field et al. 1994) and the planetary nebulae Parsamyan 18 (Sellgren 1986) and Hubble 12 (Dinerstein et al. 1988, Ramsay et al. 1993).

In XDRs, the rovibrational levels are populated by cascading from electronically excited states of $\mathrm{H}_{2}$ arising from photoelectron impact and by rovibrational excitation in direct photoelectron impacts on the lowest $v=0$ vibrational level. The resulting spectrum in a diffuse gas has been calculated by Gredel \& Dalgarno (1995) and in a dense gas by Tiné et al. (1996). However, there is no astronomical object in which X-ray pumping has been clearly identified as the excitation mechanism.

\section{Time-dependent PDRs}

Several recent theoretical studies have addressed time dependent effects in evolving $\mathrm{H} / \mathrm{H}_{2}$ dissociation fronts in PDRs (Goldshmidt \& Sternberg 1995; Hollenbach \& Natta 1995; Bertoldi \& Draine 1996). These studies are motivated by the fact that the equilibrium timescales for the development of $\mathrm{H} / \mathrm{H}_{2}$ photodissociation fronts are often long $\left(5 \times 10^{8} / n_{\mathrm{H}} \mathrm{yr}\right)$ compared to the turn-on times $\left(\sim 10^{5}\right.$ yr for OB stars, or $\leqslant 10^{3}$ yr in planetary nebulae) or lifetimes $\left(\lesssim 10^{7} \mathrm{yr}\right.$ for OB stars, or $\lesssim 10^{5} \mathrm{yr}$ in planetary nebulae) of the 
FUV radiation sources. In clumpy clouds in star-forming regions, or in the vicinity of active galactic nuclei fresh material may suddenly be exposed to intense FUV radiation fields as embedded clumps move out from within "shadowed" regions.

Goldshmidt \& Sternberg (1995) presented explicit computations and analysis of the time-varying intensities of infrared FUV-pumped fluorescent $\mathrm{H}_{2}$ emission in (low-density) PDRs. They showed that in clouds exposed to intense radiation fields the $\mathrm{H}_{2}$ emission line intensities at early times in the evolution of the PDRs are much larger than the line intensities that are produced after the $\mathrm{H} / \mathrm{H}_{2}$ photodissociation fronts reach their equilibrium configurations. In clouds exposed to intense fields the increasing dust opacity associated with the large $\mathrm{H} \mathrm{I}$ zones that form as the $\mathrm{H} / \mathrm{H}_{2}$ dissociation fronts advance into the clouds reduces the fraction of the incident FUV photons that are absorbed by the $\mathrm{H}_{2}$ molecules and converted into infrared vibrational emission.

This time-dependent behavior probably explains the unusually intense $\mathrm{H}_{2}$ line emission observed in several young planetary nebulae. For example, the average surface brightness of the $2.12 \mu \mathrm{m} \mathrm{1-0} \mathrm{S}(1) \mathrm{H}_{2}$ line $\left(10^{-3}\right.$ ergs $\left.\mathrm{s}^{-1} \mathrm{~cm}^{-2} \mathrm{sr}^{-1}\right)$ in the planetary nebula $\mathrm{BD}+30^{\circ} 3639$ is more than 100 times brighter than would be expected under conditions of equilibrium given the

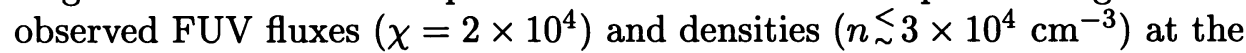
edges of the molecular shells. However, this planetary nebula is probably only $10^{3}$ yrs old (as inferred from its size and expansion velocity). At this time the predicted $\mathrm{H}_{2}$ intensity is (for the above values of $\chi$ and $n$ ) about 100 times larger than the equilibrium value.

\section{Acknowledgements}

The work of AD and MY was supported by the National Science Foundation, Division of Astronomical Sciences, under grant AST 95-31790.

\section{References}

Bertoldi, F., Draine, B.T. 1996, ApJ 458, 222

Black, J.H., Dalgarno, A. 1976, ApJ 203, 132

Black, J.H., Porter, A., Dalgarno, A. 1981, ApJ 249, 138

Black, J.H., van Dishoeck, E.F. 1987, ApJ 332, 412

Burton, M.G., Bulmer, M., Moorhouse, A., Geballe, T.R., Brand, P.W.J.L. 1992, MNRAS $257,1 P$

Dalgarno, A. 1976, in Atomic Processes and Applications eds. P.G. Burke and B.L. Moisewitsch (North Holland: Amsterdam), p. 109

Dinerstein, H.L., Lester, D.F., Carr, J.S., Harvey, P.M. 1988, ApJ 327, L 27

Draine, B.T., Bertoldi, F. 1996 ApJ 468, 269

Escalante, V., Sternberg, A., Dalgarno, A. 1991, ApJ 369, 213

Field, D., Gerin, M., Leach, S., Lemaire, J.L., Pineau des Forêts, G., Rostas, F., Roueff, E., Simons, D. 1994, A\&A 286, 909; A\&A 276, 274 
Fuente, A., Martín-Pintado, J., Cernicharo, J., Bachiller, R. 1993, A\&A 276, 473

Fuente, A., Martín-Pintado, J., Gaume, R. 1995, ApJ 442, L33

Gatley, I., Hasegawa, T, Suzuki, H., Garden, R., Brand, P., Lightfoot, J. Glencross, W., Okuda, H., Nagata, T., 1987, ApJ 318, L73

Genzel, R., Harris, A.J., Jaffe, D.T., Stutzki, J. 1988, ApJ 332, 1049

Goldshmidt, A., Sternberg, A. 1995, ApJ 439, 256

Gould, R.J., Harwit, M. 1963, ApJ 137, 694

Gredel, R., Dalgarno A. 1995, ApJ 446, 852

Hartquist, T.W., Rawlings, J.M.C., Williams, D.A., Dalgarno, A. 1993, Quart. J. R. Astron. Soc. 34, 213

Hasegawa, T., Gatley, I., Garden, R.P., Brand, P.W.J.L., Ohishi, M., Hayashi, M, Kaifu, N. 1987, ApJ 318, L77

Hollenbach, D., Natta, A. 1995, ApJ 455, 133

Hollenbach, D.J., Takahashi, T., Tielens, A.G.G.M. 1991, ApJ 377, 192

Jansen, D.J., van Dishoeck, E.F., Black, J.H., Spaans, M., Sosin, C. 1995, A\&A 302, 223

Köster, B. Störzer, H., Stutzki, J., Sternberg, A. 1994, A\&A 284, 545

Keene, J., Blake, G.A., Phillips, T.G., Huggins, P.J., Beichman, 1985, ApJ 299, 967

Krolik, J.H., Kallman, T.R. 1983, ApJ 267, 610

Langer, W.D. 1978, ApJ 225, 800

Latter, W.B., Walker, C.R., Maloney, P.R. 1995, ApJ 419, L97

Le Bourlot, J., Pineau des Forêts, G., Roueff, E., Flower, D.R. 1993, A\&A 267, 233

Lepp, S., Dalgarno, A. 1996, A\&A 306, L21

Lepp, S., McCray, R. 1983, ApJ 269, 560

Luhman, M.L., Jaffe, D.T. 1996, ApJ 463, 191

Luhman, M.L., Rieke, G.H. 1996, ApJ 461, 298

Maloney, P.R., Hollenbach, D.J., Tielens, A.G.G.M. 1996, ApJ 466, 561

McKee, C.F. 1989, ApJ 345, 782

Meixner, M., Haas, H.R., Tielens, A.G.G.M., Erickson, E.F., Werner M. 1992, ApJ 390, 499

Meixner, M., Tielens, A.G.G.M. 1993, ApJ 405, 216

Meixner, M., Tielens, A.G.G.M. 1995, ApJ 446, 907

Morrison, R., McCammon, D. 1983, ApJ 270, 119

Natta, A., Walmsley, C.M., Tielens, A.G.G.M. 1994, ApJ 438, 209

Neufeld, D.A., Dalgarno, A. 1989, ApJ 344, 251; 340, 869

Neufeld, D.A., Maloney, P.R. 1995, ApJ 447, L17

Neufeld, D.A., Maloney, P.R., Couger, S. 1994, ApJ 436, L127

Neufeld, D.A., Spaans, M. 1996, ApJ in press

Phillips, J.A, Lazio, J.W. 1995, ApJ 442, L37

Phillips, T.G., Huggins, P.J. 1981, ApJ 251, 533

Prasad, S.S., Tarafdar, S.P. 1983, ApJ 267, 603

Ramsay, S.K., Crysostomou, A., Geballe, T.R., Brand, P.W.J., Mountain, M. 1993, MNRAS 263, 695

Roberge, W.G., Dalgarno, A. 1982, ApJ 255, 480

Schilke, P., Carlstrom, J.E., Keene, J., Phillips, T.G. 1993, 417, L67

Sellgren, K. 1986, ApJ 305, 399

Störzer, H., Stutzki, J., Sternberg, A., 1995, A\&A 296, L9

Störzer, H., Stutzki, J., Sternberg, A., 1996, A\&A submitted

Stacey, G.J., Jaffe, D.T., Geis, N., Genzel, R., Harris, A.I., Poglitsch, A., Stutzki, J., Townes, C.H. 1993, ApJ 404, 219

Sternberg, A. 1988, ApJ 332, 400

Sternberg, A., Dalgarno, A. 1989, ApJ 338, 197

Sternberg, A., Dalgarno, A. 1995, ApJS 99, 565

Stutzki, J., Stacey, G.J., Genzel, R., Harris, A.J., Jaffe, D.T., Lugten, J.B. 1988, ApJ 332,379

Tauber, J.A., Lis, D.C., Keene, J., Schilke, P. , Büttgenbach, T.H. 1995, A\&A 297, 567 
Tielens, A.G.G.M., Hollenbach, D. 1985, ApJ 291, 722 and 747

Tiné, S., Gredel, R., Lepp, S., Dalgarno, A. 1996, ApJ submitted

Turner, B.E. 1992, ApJ 396, L107

Turner, B.E. 1994, ApJ 430, 727

van der Werf, P.P., Genzel, R., Krabbe, A., Blietz, M., Lutz, D., Drapatz, S., Ward, M.J., Forbes, D.A. 1993, ApJ 405, 522

van der Werf, P.P., Stutzki, J., Sternberg, A., Krabbe, A. 1996, A\&A 313, 633

van Dishoeck, E.F., Black, J.H. 1988, ApJ 334, 771

Weisheit, J.C. 1974, ApJ 190, 735

Weisheit, J.C., Dalgarno, A. 1972, Ap. Lett. 12, 105

Wolfire, M.G., Königl, A. 1993, ApJ 415, 204

Yan, M., Dalgarno, A. 1996a, ApJ to be submitted

Yan, M., Dalgarno, A. 1996b, ApJ in press

Zhou, S., Jaffe, D.T., Howe, J.E., Geis, N., Herrmann, F., Madden, S.C., Poglitsch, A., Stacey, G.J. 1993, ApJ 419, 190

Zmuidzinas, J., Betz, A.L., Boreiko, T.R., Goldhaber, D.M. 1988, ApJ 335, 774

\section{Discussion}

Greenberg: Couldn't NH form by reactions on grain surfaces? For typical UV radiation fields, I believe that the dust temperature must be about $70 \mathrm{~K}$. Does this high dust temperature help or hinder?

Dalgarno: Grain surface reactions could be a source of NH. It is usually argued that for diffuse clouds they are such a source. I do not know how they might be modified by a temperature of $70 \mathrm{~K}$.

Fuente: Just a comment. We have detected $\mathrm{CO}^{+}$toward the reflection nebula NGC 7023. The results are shown in a poster. 


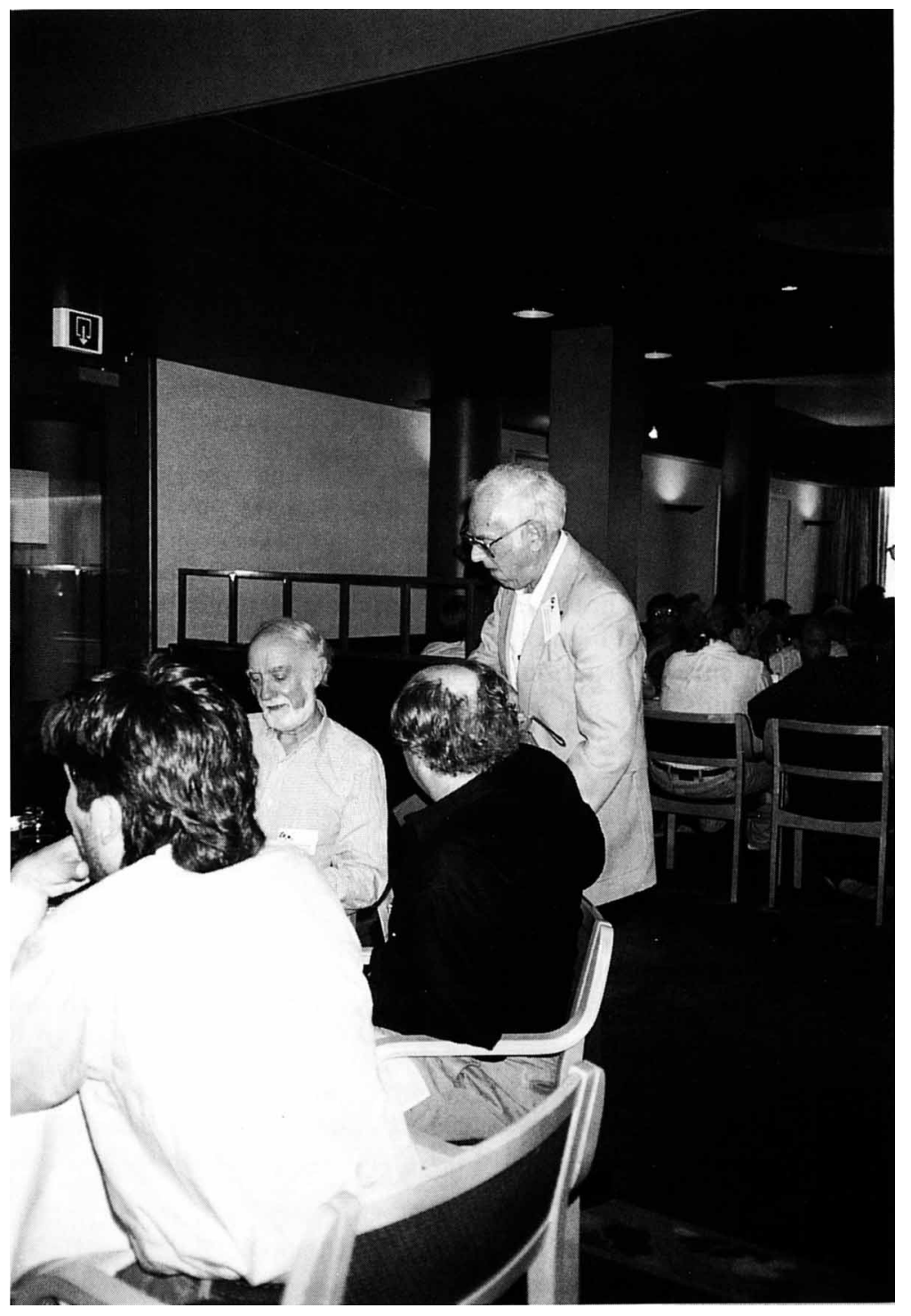

